\title{
Some Reflections on Model Predictive Control of Transmission Voltages
}

\author{
Mevludin Glavic, Member, IEEE, Thierry Van Cutsem, Fellow, IEEE
}

\begin{abstract}
This paper deals with the application of algorithms inspired by Model Predictive Control to solve voltage-related power system control problems in both normal and emergency operating conditions. In the first part of the paper, we identify critical issues for a practical implementation of this methodology, and analyze how far these requirements have been met so far. In the second part, we outline a voltage control scheme that hopefully addresses the above issues. The central idea of this scheme is a static optimization to determine target control values, followed by a dynamic optimization to produce a feasible transition, both carried out in the closed-loop mode of Model Predictive Control.
\end{abstract}

Index Terms - Model predictive control, quasi steady-state model, optimization, voltage control, secondary voltage control.

\section{INTRODUCTION}

$\mathrm{D}^{\mathrm{s}}$ ESIGNERS of power system controls have been always concerned first and foremost with safety and reliability. Only those control techniques that are fully understood, matured, and are guaranteed to work are used [1].

Model Predictive Control (MPC) techniques are both mature (being considered a viable control strategy, they have been used for years in process industry [2,3]) and theoretically understood (see [4,5] for a good account on the main theoretical results). It is thus somewhat surprising that up to now this control technique has not received more attention from power system researchers and practitioners, although recent references show a growing interest for this approach [6-21].

Practical interest in using MPC is driven by the fact that industrial processes need to be operated under tighter performance specifications and at the same time more and more constraints (dictated by economical, environmental, and safety considerations) need to be satisfied [2,3]. This fact is, more than ever, present in today's power systems. Precisely, the ability of MPC to incorporate various constraints makes it attractive in this respect. Obviously, one could also quote the growth in computational power that allows performing fasterthan-real-time simulations, as well as the availability of proven models and efficient optimization algorithms.

Mevludin Glavic (glavic@montefiore.ulg.ac.be) is an invited professor of the University of Liège, Dept. of Electrical Engineering and Computer Science, Sart Tilman B28, B-4000 Liège, Belgium. Thierry Van Cutsem (t.vancutsem@ulg.ac.be) is with FNRS (Belgian Funds for Scientific Research) at the same department.
Some achievements on using MPC for system frequency control [18-20] and power plant control [21] problems have been reported. This paper, however, focuses on the control of transmission system voltages.

We first discuss what we believe are critical issues for the application of MPC techniques to voltage control problems. For each issue, we briefly review some of the proposed approaches [6-17] and possibly identify some further research needs.

Next, we outline a new MPC voltage control scheme for normal and emergency system conditions that meets the previously identified requirements. The control strategy is made up of two parts: a static optimization to determine moving target controls, followed by a dynamic optimization to produce a feasible transition. Both are repeated at each time step according to the closed-loop structure of MPC. This scheme offers some similarities with the so-called coordinated secondary voltage control scheme, presently in operation in part of the French transmission system.

The paper is organized as follows. Section II recalls the standard MPC formulation. Section III addresses some of the critical issues for MPC application to voltage problems, and briefly surveys the proposed approaches. Section IV outlines a new MPC voltage control scheme while Section V offers some conclusions.

\section{Brief Review of Modal Predictive Control}

Standard MPC (also referred to as moving horizon or receding horizon control) is essentially a class of computer control algorithms to control the future behaviour of a system through the use of an explicit model of the latter [4,5]. At each control step the MPC algorithm computes an open-loop sequence of controls in order to optimize the future system behaviour. The first control action in the optimal sequence is applied and the entire optimization is repeated at subsequent control steps. The principle of MPC is illustrated in Fig. 1.

It is convenient to formulate the MPC problem in the context of a discrete-time, nonlinear system described by [3]:

$$
\begin{aligned}
& \mathbf{x}_{k+1}=\mathbf{f}\left(\mathbf{x}_{k}, \mathbf{u}_{k}, \mathbf{v}_{k}\right) \\
& \mathbf{y}_{k}=\mathbf{g}\left(\mathbf{x}_{k}\right)+\mathbf{w}_{k}
\end{aligned}
$$

where $\mathbf{x}_{k}$ is a vector of state variables, $\mathbf{u}_{k}$ a vector of controls, $\mathbf{y}_{k}$ a vector of outputs or controlled variables, $\mathbf{v}_{k}$ 
and $\mathbf{w}_{k}$ are noise vectors.

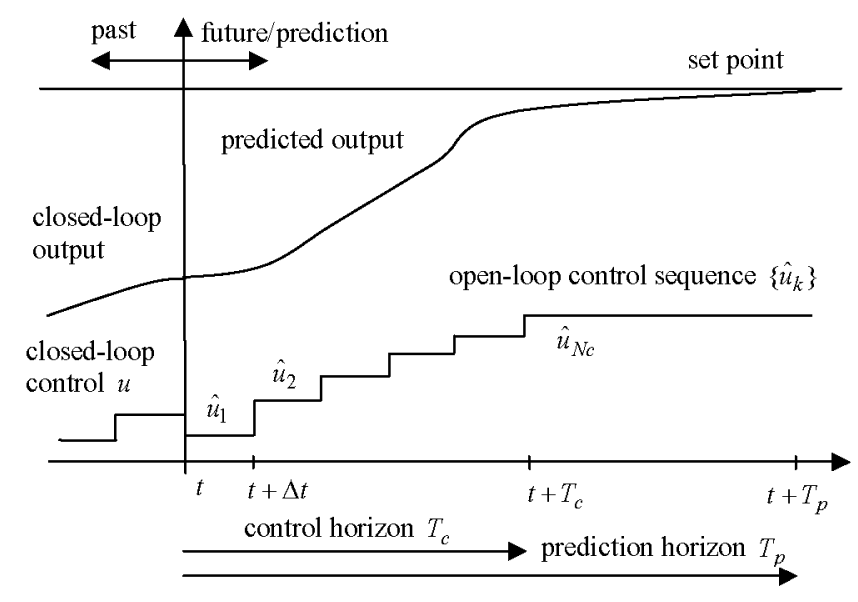

Fig. 1. Principle of MPC

The problem is to compute a sequence of controls $\left\{\mathbf{u}_{k}\right\} \quad\left(k=0, \ldots, N_{c}-1\right)$ that will take the system from its current state $\mathbf{x}_{k}$ to a desired steady state $\mathbf{x}_{s}$. The desired steady state $\left(\mathbf{x}_{s}, \mathbf{u}_{s}, \mathbf{y}_{s}\right)$ is either fixed or determined by a steady-state optimization. In the latter case, it can be computed either once for all or at each time step.

The MPC algorithm consists in minimizing the objective function:

$$
\begin{array}{r}
J=\quad \sum_{j=1}^{N p}\left\|\mathbf{y}_{k+j}-\mathbf{y}_{s}\right\|_{\mathbf{Q}}^{2}+\sum_{j=0}^{N c-1}\left\|\mathbf{u}_{k+j}-\mathbf{u}_{s}\right\|_{\mathbf{R}}^{2}+ \\
\sum_{j=0}^{N c-1}\left\|\mathbf{u}_{k+j}-\mathbf{u}_{k+j-1}\right\|_{\mathbf{S}}^{2}+\|\mathbf{S}\|_{\mathbf{T}}^{2}
\end{array}
$$

subject to the constraints:

$$
\begin{array}{cc}
\mathbf{x}_{k+j}=\mathbf{f}\left(\mathbf{x}_{k+j-1}, \mathbf{u}_{k+j-1}\right) & j=1, \ldots, N_{p} \\
\mathbf{y}_{k+j}=\mathbf{g}\left(\mathbf{x}_{k+j}\right) & j=1, \ldots, N_{p} \\
\underline{\mathbf{y}}-\mathbf{s} \leq \mathbf{y}_{k+j} \leq \overline{\mathbf{y}}+\mathbf{s} & j=1, \ldots, N_{p} \\
\underline{\mathbf{u}} \leq \mathbf{u}_{k+j} \leq \overline{\mathbf{u}} & j=1, \ldots, N_{c}-1 \\
\underline{\boldsymbol{\delta}} \leq \mathbf{u}_{k+j+1}-\mathbf{u}_{k+j} \leq \overline{\boldsymbol{\delta}} & j=1, \ldots, N_{c}-1 \\
\mathbf{s} \geq \mathbf{0} &
\end{array}
$$

The first three terms in the objective function (2) aim at penalizing future deviations of outputs from the desired steady state over the prediction horizon $T_{p}\left(N_{p}=T_{p} / \Delta t\right)$, future deviations of controls from the desired steady-state over the control horizon $T_{c}\left(N_{c}=T_{c} / \Delta t\right)$, and future rapid changes in controls, respectively. The last term in (2) is used to minimize the magnitude of output constraint violations. The weighting matrices $\mathbf{Q}, \mathbf{R}, \mathbf{S}$ and $\mathbf{T}$ are chosen positive definite. Other constraints than those shown in (4) may be considered as well. The weighting matrices and the bounds have been assumed constant for simplicity.

An efficient implementation of MPC requires the following issues to be addressed first [2-5]:

1. system model: it should be both accurate and computationally tractable. To keep the problem tractable, one or several successive system linearizations are usually considered [2,3];

2. sampling time, prediction and control horizons: the sampling interval basically depends on the system dynamics. The prediction horizon $T_{p}$ should be chosen long enough so as to cover the system settling time. The control horizon $T_{c}$ is usually smaller than the prediction horizon and represents the number of possible control moves. Short horizons are desirable from the computational point of view, but long horizons are required for closed-loop stability and in order to achieve the desired performance in closed-loop;

3. optimization method: with the quadratic objective function (2) and a linearized system model, the resulting optimization takes on the form of a highly structured convex Quadratic Programming (QP) problem. Several standard solution algorithms and codes are available for this problem [2-5].

\section{CRitical issues in MPC apPlication to voltage PROBLEMS}

\section{A. Overview of proposed approaches}

References [6-17,22,23] make up a representative (although certainly not exhaustive) sample of publications devoted to application of MPC to voltage-related problems.

Expectedly, the proposed approaches do not all strictly adhere to the original MPC algorithm, but at least all of them share two major ingredients of MPC: prediction and optimization. Among the "MPC-like" schemes, some of them differ from the basic MPC algorithm in that they minimize a given cost function by selecting the optimal sequence of control actions, among available ones, and evaluate them online, using the system model for performance prediction. This combinatorial search is pointed out in Table 1, together with some other characteristics.

\section{B. Objectives and control means}

In normal operating conditions the objective is to keep network voltages as close as possible to reference values. To this purpose synchronous generators are the components it is most natural to act on. It is also appropriate to control the system in such a way that generators with a larger reactive power capability participate more ("fair" sharing of reactive effort). Depending of the system specificities, a variety of other control means can be used [6-8,10-11,14-17]: synchronous condenser and static var compensator voltages, (capacitive or inductive) shunt compensation, voltage 
setpoints of more advanced FACTS devices, voltage setpoints of Load Tap Changing (LTC) transformers.

TABLE I

SAMPLE OF MPC APPROACHES TO VOLTAGE-RELATED PROBLEMS

\begin{tabular}{|c|c|c|c|}
\hline Problem & Model & $\begin{array}{c}\text { Optimization } \\
\text { method }\end{array}$ & Combinatorial? \\
\hline $\begin{array}{c}\text { Emergency } \\
\text { voltage } \\
\text { control }\end{array}$ & $\begin{array}{c}\text { Linear } \\
{[9-11,13-17]} \\
\text { Nonlinear } \\
{[6-8,12]}\end{array}$ & $\begin{array}{c}\text { Tree search [6], } \\
\text { Branch-and-bound } \\
{[11,17],} \\
\text { Pseudo-gradient } \\
\text { evolutionary } \\
\text { programming [8], } \\
\text { QP and branch-and- } \\
\text { bound [10,14], } \\
\text { Linear programming } \\
{[9,13],} \\
\text { Mixed integer } \\
\text { programming } \\
{[15,16] .}\end{array}$ & $\begin{array}{l}\text { yes } \\
\text { yes } \\
\text { yes } \\
\text { yes } \\
\text { no } \\
\text { yes }\end{array}$ \\
\hline $\begin{array}{l}\text { Design of } \\
\text { system } \\
\text { protection } \\
\text { scheme }\end{array}$ & Linear & Heuristic tree search & yes \\
\hline $\begin{array}{l}\text { Secondary } \\
\text { voltage } \\
\text { control }\end{array}$ & Linear & $\begin{array}{l}\text { Quadratic } \\
\text { programming }\end{array}$ & no \\
\hline
\end{tabular}

The main expected advantage of MPC in normal operating conditions is the coordinated control of the various control means. Such a scheme would relieve the control center operators from the heavy and possibly delicate task of successively adjusting numerous controls.

As far as MPC is concerned, different variants of the standard quadratic objective function (2) have been used. In several cases, the objective includes all but the second term $[6-8,12]$. As a variant of the fourth term, a penalty can be introduced whenever a constraint violation or a singularityinduced bifurcation is predicted to occur within a prediction horizon. In [8] a penalty term has been also added to account for constraint violations and instability. The objective functions of [14-17] include the first three terms of (2). Some publications penalize the amount of control at each sampling time but no publication includes the second term of (2) which penalizes future deviations of controls from the desired steady-state.

To some extent, the above voltage control task is performed automatically by Secondary Voltage Control (SVC). The first generation of SVC, in operation in France and Italy, relies on reactive power control loops in power plants and a centralized PI controller to regulate the voltage at a pilot node (whose voltage is representative of the other bus voltages in the area of concern), while sharing the effort over the participating generators according to predefined participation factors $[24$, 25].

Interestingly, the second generation of SVC [22], in operation in some parts of the French system, can be seen as a special implementation of the MPC concept, in so far as it relies on the multi-step optimization of a quadratic voltagereactive power objective, embedding new measurements of pilot node voltages and generator reactive powers at each time step. It can be said that this is the only voltage control scheme bearing some features of MPC and in real operation today.

In emergency conditions, where voltages undergo unacceptable deviations from their reference values, or even become unstable, the objective is essentially the same; the main difference is the higher speed and magnitude of actions needed. It is well-known that higher source voltages yield higher load power margins and a quick increase of generator voltages may contribute to stabilizing a system, or at least to reducing the size of more drastic countermeasures. Furthermore, control means may include load shedding in the last resort. Where system integrity is endangered, corrective actions may be taken at the expense of some sub-optimality, the priority being to save the largest possible part of the system.

The main expected advantage of MPC in emergency operating conditions is again the coordinated control of the various control means. This is even more crucial since operators may be working under stress and the system is close to or outside its normal limits making the control task even more delicate. To our best knowledge, the present SVC schemes do not have a particular emergency mode. We believe that emergency voltage control is the area where an efficient MPC algorithm could prove most useful.

Load shedding has been considered as a control means by several authors either together with other controls [6-17] or alone $[9,13]$. For instance, a non-disruptive load control scheme has been considered in [9,13], as a hierarchical control structure where an MPC controller acts at the upper level and coordinates lower (substation) level controllers.

In theory, an MPC scheme would benefit from a wide view of the system operating state, and could be at the heart of a wide area control system, allowing for instance to better adjust the amplitude and location of load shedding to the disturbance of concern. However, the shedding of loads through an MPC scheme may be questioned by practitioners. Indeed, even in the case of long-term voltage stability it may be required to shed load within seconds after the disturbance occurrence [26] and it is not clear whether an MPC scheme would be fast enough. Moreover, in such a system protection scheme, preference is given to simplicity for reliability reasons. The many components that enter an MPC-driven load shedding scheme increase the probability of failure (i.e. decrease the protection reliability) while local undervoltage load shedding controllers make up a much simpler alternative.

\section{System modelling}

The response time of an MPC-based voltage control ranging from - say - 10 to 60 seconds, long-term dynamics are of concern. In this context, it is appropriate to resort to the Quasi-Steady State (QSS) approximation of the long-term dynamics [27]. The essence of this method is time-scale decomposition, faster phenomena being represented by their equilibrium conditions instead of their full dynamics.

Most of the listed publications resort more or less implicitly 
to this technique, which greatly reduces the complexity of the resulting model and hence provides the computational efficiency required to meet the constraints of an on-line application. Moreover, the amount of additional data required by the QSS model is moderate, so that data collection, validation and maintenance are not a big issue.

The QSS model takes on the form:

$$
\begin{gathered}
\mathbf{0}=\mathbf{g}\left(\mathbf{x}_{1}, \mathbf{x}_{2}, \mathbf{y}, \mathbf{z}\right) \\
\mathbf{0}=\mathbf{f}_{1}\left(\mathbf{x}_{1}, \mathbf{x}_{2}, \mathbf{y}, \mathbf{z}\right) \\
\dot{\mathbf{x}}_{2}=\mathbf{f}_{2}\left(\mathbf{x}_{1}, \mathbf{x}_{2}, \mathbf{y}, \mathbf{z}\right) \\
\mathbf{z}\left(t_{k}^{+}\right)=\mathbf{h}\left(\mathbf{x}_{1}, \mathbf{x}_{2}, \mathbf{y}, \mathbf{z}\left(t_{k}^{-}\right)\right)
\end{gathered}
$$

where Eq. (5) stands for the network equations ( $y$ being the vector of bus voltages), Eq. (6) for the short-term dynamics replaced by equilibrium conditions ( $\mathbf{x}_{1}$ being the corresponding algebraic variables), Eq. (7) for the continuous longterm dynamics (corresponding state vector $\mathbf{x}_{2}$ ) and Eq. (8) captures discrete events stemming from controllers (e.g. LTCs), protections (e.g. OverExcitation Limiters (OELs)) and possibly system protection schemes (e.g. load shedding if taken care of by local controllers), causing the variables $\mathbf{z}$ to undergo step changes at some times $t_{k}$. Note that when the long-term dynamics are driven by LTCs and OELs only, the QSS system trajectory amounts to a succession of short-term equilibria, each being the solution of Eqs. $(5,6)$ and the change from one equilibrium to the next being dictated by (8). The interested reader may refer to $[27,28]$ for a more detailed description of the above model, as well as some extensions of the QSS technique.

QSS simulation is very fast and compatible with the on-line requirements. For instance, it takes less than 2 seconds to simulate the 15-minute response of a 1000-bus system to a large disturbance [28]. The technique thus offers the possibility to evaluate the system response much faster than real-time. Following a large disturbance, assuming that the change in topology can be identified, it is quite feasible to anticipate the system behaviour from a QSS simulation of the disturbance, initialized from the pre-disturbance conditions.

The same fast technique could also be used to determine the controls to apply to regulate network voltages. However, models are always approximations, especially those involving the uncertain load behaviour; therefore, it may not be acceptable, especially in emergency conditions to apply controls determined from the sole QSS model. The motivation for using MPC is precisely to compensate for those model inaccuracies, by implementing the controls in a closed-loop manner, involving measured gathered from the system.

Besides MPC itself, it remains of interest to set up a technique to correct the QSS model from the discrepancies observed between the measured and the simulated system evolutions. This appears, however, to be a very challenging problem. For the time being, it appears more reasonable to restrict the use of the faster-than-real-time QSS simulation to the early detection of a situation evolving to emergency conditions. This may be used to trigger an emergency mode in the algorithms.

\section{Trajectory prediction}

The prediction of the future system behaviour is an important part of any MPC algorithm. However, the nonlinear model (5-8) cannot be easily used to this purpose. As mentioned previously, it is required to resort to some sort of model simplification. Among the main techniques used to this purpose let us quote [6]:

1. Euler state prediction $[6-8,12]$, which has been used together with an approximation of the output trajectories by one straight line between their values at the beginning and the end of the prediction interval [6-8]. Euler state prediction applies to Eq. (7) of the QSS model;

2. off-equilibrium dynamic linearization [6,14], which consists of a classical linearization of Eqs. (5-7) performed at the current operating point (which is generally not an equilibrium). This linearization can be performed numerically [6] or symbolically [14];

3. trajectory sensitivities $[9,11,12,17]$ which provide a systematic way to compute sensitivities of the trajectory of $x_{2}$ (Eq. (7)) with respect to changes in parameters, initial conditions, and structural changes. The approach is based on linearizing the system model around a nominal trajectory [30] rather than an equilibrium point. Therefore it is possible to quantify the variation of a trajectory resulting from a (small) change in parameters, and/or initial conditions, and /or structural changes.

We note incidentally that very few references take into account the discrete nature of the transitions captured by Eq. (8), which are the only long-term dynamics for a system driven by LTCs and OELs.

More importantly, it seems that most approaches rely on the knowledge of the whole system state in order to predict its future evolution. This requires including the EMS state estimator in the MPC loop, which is not desirable as far as the convergence, unobservability and bad data problems it may experience, especially in emergency operating conditions, would impair the reliability of the MPC scheme. On the contrary, an MPC scheme that relies on dedicated measurements and can operate without knowing the remaining of the system state vector appears as a highly desirable feature, even if the price to pay is a decrease in model accuracy.

\section{E. Combinatorial handling of discrete controls}

The fact that some controls (e.g. LTCs or shunt compensation) are discrete by nature has led some authors to adopt at least a hybrid, if not a fully discrete formulation (considering that some continuous controls can be reasonably discretized). Expectedly, the large number of controls available in a real-life power system, together with their combination at successive time steps leads to highly combinatorial problems. This implies in practice that a huge 
number of system responses would have to be evaluated online. For instance, for a simple 6-bus system [6-8] with 8 available controls as many as 2,000 online evaluations of the system evolution were envisaged in order to determine the control sequence!

The discrete nature of the problem also motivated some authors to use a hybrid approach based on mixed logical dynamic framework $[11,15,16]$. Although elegant, this approach seems to be even more computationally demanding.

Methods that cut down the combinatorial explosion are thus essential. In this context, Refs. [6-8,12] proposed to resort to heuristic or meta-heuristic optimization methods while in $[11,17]$ the problem was tackled using the branchand-bound method. The same optimization method was used in [14] to solve the discrete control part of the problem, together with QP for continuous controls. In $[15,16]$ a mixed integer programming method has been used with the help of CPLEX software environment.

Some figures about the complexity of reported tests are given in Table II. It reveals that only small-size systems have been used so far to demonstrate effectiveness of MPC techniques. The average time taken by the optimization method in [8] is $61.1 \mathrm{~s}$ (using simple Euler state predictor). The authors hope that this computing time can be improved by better software implementation [8]. However, the viability of the method has still to be checked on real-life systems. The average computing times reported in [16] for the optimization are in the order of $1.8 \mathrm{~s}$ for 4 prediction steps and $160 \mathrm{~s}$ for 7 prediction steps. Again, these times relate to a very small, 4bus test system $[15,16]$.

Some combinatorial approaches seem motivated by the search of a globally optimal control sequence. In both normal and emergency conditions, this quest for optimality seems somewhat utopian insofar as the objective considered here is more technical than economical (at least as long the market does not put financial penalties on voltage deviations!).

TABLE II

COMPLEXITY FIGURES OF REPORTED TESTS

\begin{tabular}{c|c|c}
\hline \hline & Test system & $\begin{array}{c}\text { Prediction horizon/ } \\
\text { sampling }\end{array}$ \\
\hline & 4-bus [10,16] & $30-150 \mathrm{~s} / 30 \mathrm{~s}[10]$ \\
Emergency voltage & 6-bus [6,8] & $120 \mathrm{~s} / 30 \mathrm{~s}, 70 \mathrm{~s} / 10 \mathrm{~s}[16]$ \\
control & 9 -bus [14] & $120 \mathrm{~s} / 30 \mathrm{~s}[6]$ \\
& 10 -bus [9,13] & $10 \mathrm{~s} / 5 \mathrm{~s}[11,17]$ \\
& 12 -bus [15] & $60 \mathrm{~s} / 30 \mathrm{~s}[8]$ \\
& 32-bus [7,11,17] & $50 \mathrm{~s} / 50 \mathrm{~s}[9,13]$ \\
& $39-$-bus [8] & $90 \mathrm{~s} / 30 \mathrm{~s}[14]$ \\
\hline Design of system & & \\
protection scheme & $32-$ bus & $120 \mathrm{~s} / 30 \mathrm{~s}[7]$ \\
against voltage & & \\
collapse & & \\
\hline \multicolumn{2}{|c|}{}
\end{tabular}

\section{OUTLINING A REALISTIC MPC CONTROL SCHEME}

We outline hereafter a voltage control scheme inspired of the MPC methodology and satisfying the following requirements, stemming from the considerations of Section III:

- leave load shedding to a distinct system protection scheme and concentrate on generator voltages and shunt compensation as controls. LTC setpoints could be included as well but are not considered here for simplicity

- avoid combinatorial optimization for efficiency reasons; instead, treat discrete controls in a continuous way

- use a relatively simple sensitivity model to anticipate the future system behaviour, counting on the closed-loop nature of MPC to compensate for those simplifications

- derive the corresponding sensitivities through formulae exploiting the sparsity of standard power system models

- avoid relying on the EMS state estimator inside the MPC control loop

- rely instead on dedicated measurements relative to selected bus voltages and to the above listed controls

- allow faster responses if emergency conditions are detected.

The proposed scheme is to some extent inspired of the previously mentioned French coordinated secondary voltage control [22]. However, it differs from the latter by its expected ability to respond more quickly in more severe situations and by the way the future evolution is optimized. In this respect, the scheme of [22] does not involve multiple prediction steps into the optimization but rather corrects a fraction of the voltage deviations at each time step.

The proposed scheme has also some similarities with the flexible coordinated secondary voltage control introduced in [23], from which it differs, however, in both the static and the dynamic optimization sub-problems (an optimal unbiased Kalman predictor has been used in [23]) as well as implementation and modeling details.

\section{A. Required information and model}

We re-use the idea, implemented in Secondary voltage control, of monitoring the transmission voltages at pilot nodes. The main motivation is to limit the number of bus voltage measurements that have to be telemetered and processed centrally. The voltage at a pilot node is assumed to be representative of the voltages in a whole area surrounding this bus. Let $p$ be the number of pilot nodes and $\mathbf{v}_{p}$ the vector of pilot node voltages.

Let $g$ be the number of generators whose voltages are controlled, $\mathbf{v}_{g}$ the corresponding vector of terminal voltages and $\mathbf{q}_{g}$ the one of reactive power productions.

Let $c$ be the number of buses where shunt compensation can be adjusted and $\mathbf{b}_{c}$ the corresponding vector of shunt admittances.

We define the following vectors of setpoint values: $\mathbf{v}_{p}^{\text {ref }}$ for pilot node voltages, $\mathbf{q}_{g}^{\text {ref }}$ for generator reactive powers and $\mathbf{b}_{c}^{\text {ref }}$ for shunt compensation. $\mathbf{v}_{p}^{\text {ref }}$ can be determined by an Optimal Power Flow aimed at minimizing the system power 
losses [22]. The choice of $\mathbf{q}_{\mathrm{g}}^{\text {ref }}$ and $\mathbf{b}_{c}^{\text {ref }}$ will be discussed in the sequel. At step $k$, we assume that pilot node voltage measurements and reactive power generation measurements are made available to the MPC algorithm in respectively $\mathbf{v}_{p}(k)$ and $\mathbf{q}_{g}(k)$. We also assume that the status of shunt devices is known, which is translated into a vector of "measured" shunt admittances $\mathbf{b}_{c}(k)$.

\section{B. Sensitivity-based trajectory prediction}

As already mentioned, a linear approximation of the future system behaviour is desirable for incorporation in the overall MPC optimization. We propose to derive the corresponding sensitivities from the system conditions at equilibrium. In other words, the sensitivities will indicate how much the steady-state values of pilot node voltages and generator reactive powers change with generator voltages and shunt admittances.

We thus consider the QSS model (5-8) at a long-term equilibrium:

$$
\begin{aligned}
& \mathbf{0}=\mathbf{g}\left(\mathbf{x}_{1}, \mathbf{x}_{2}, \mathbf{y}, \mathbf{z}\right) \\
& \mathbf{0}=\mathbf{f}_{1}\left(\mathbf{x}_{1}, \mathbf{x}_{2}, \mathbf{y}, \mathbf{z}\right) \\
& \mathbf{0}=\mathbf{f}_{2}\left(\mathbf{x}_{1}, \mathbf{x}_{2}, \mathbf{y}, \mathbf{z}\right) \\
& \mathbf{0}=\mathbf{h}_{e}\left(\mathbf{x}_{1}, \mathbf{x}_{2}, \mathbf{y}, \mathbf{z}\right)
\end{aligned}
$$

where the last equation stands for the equilibrium conditions of the discrete devices. For a non-limited LTC, for instance, this equation takes on the simple form:

$$
V_{\text {cont }}=V_{\text {cont }}^{0}
$$

where $V_{c o n t}$ is the voltage controlled by the LTC and $V_{c o n t}^{0}$ the corresponding setpoint (midpoint of LTC deadband).

The above equations can be rewritten in compact form as:

$$
\mathbf{f}(\mathbf{x}, \mathbf{u})=\mathbf{0}
$$

where $\mathbf{u}$ is the control vector of generator voltages and shunt admittances and $\mathbf{x}$ an augmented (algebraic) state vector. Let $\eta$ be either a pilot node voltage or a generator reactive power. The sensitivity of $\eta$ with respect to $\mathbf{u}$ is given by [31]:

$$
S_{\eta \mathbf{u}}=-\mathbf{f}_{\mathbf{u}}^{T}\left(\mathbf{f}_{\mathbf{x}}^{T}\right)^{-1} \nabla_{\mathbf{x}} \eta
$$

where $\mathbf{f}_{\mathbf{u}}$ (resp. $\mathbf{f}_{\mathbf{x}}$ ) is the Jacobian matrix of $\mathbf{f}$ with respect to $\mathbf{u}$ (resp. $\mathbf{x}$ ) and $\nabla_{\mathrm{x}} \eta$ is the gradient of $\eta$ with respect to $\mathbf{x}$. The well-known formula (11) has been used in many voltage stability studies [27, 31].

There from one easily derives the linear model:

$$
\begin{aligned}
\Delta \mathbf{v}_{p} & =\mathbf{S}_{p g} \Delta \mathbf{v}_{g}+\mathbf{S}_{p c} \Delta \mathbf{b}_{c} \\
\Delta \mathbf{q}_{g} & =\mathbf{S}_{g g} \Delta \mathbf{v}_{g}+\mathbf{S}_{g c} \Delta \mathbf{b}_{c}
\end{aligned}
$$

in which $\Delta$ denotes small variations and the various matrices are assembled row by row, each row being obtained from (11).

An important aspect is the update of the above matrices We propose to evaluate them when the system is in (almost) steady state and keep them constant as long as operating conditions do not change significantly. Note that Eq. (11) requires knowing the whole system state and hence relying on the network voltages provided by the EMS state estimator.

When a disturbance occurs, such as a line or a generator outage, the sensitivity matrices must be updated. Now, it is precisely over the post-disturbance time interval that voltage control is needed. In order not to rely on the state estimator output in that period of time, it is proposed to solve the equilibrium equations (9) for the new topology (this is equivalent to the standard contingency evaluation) and recompute the sensitivity matrices at the new operating point. When the real system settles down at a new steady state, the matrices can be refreshed using the network voltages provided by the state estimator.

The following two optimizations are carried out at each time step $k$.

\section{First step: determining target controls}

The objective is to determine what would be the best controls $\Delta \mathbf{v}_{g}^{*}$ and $\Delta \mathbf{b}_{c}^{*}$ to apply in a (fictitious) single step, based on the available sensitivity information and taking into account a number of constraints.

To this purpose, the following objective function is minimized:

$$
\begin{array}{r}
J_{1}=\left\|\mathbf{v}_{p}^{r e f}-\mathbf{v}_{p}(k)-\mathbf{S}_{p g} \Delta \mathbf{v}_{g}-\mathbf{S}_{p c} \Delta \mathbf{b}_{c}\right\|_{\mathbf{P}}^{2}+ \\
\left\|\mathbf{q}_{g}^{r e f}-\mathbf{q}_{g}(k)-\mathbf{S}_{g g} \Delta \mathbf{v}_{g}-\mathbf{S}_{g c} \Delta \mathbf{b}_{c}\right\|_{\mathbf{G}}^{2}+ \\
\left\|\mathbf{b}_{c}^{r e f}-\mathbf{b}_{c}\right\|_{\mathbf{C}}^{2}
\end{array}
$$

with respect to $\Delta \mathbf{v}_{g}$ and $\Delta \mathbf{b}_{c}$, taking into account the following constraints:

- generator voltages in acceptable operating ranges:

$$
\mathbf{v}_{g}^{\min } \leq \mathbf{v}_{g}(k)+\Delta \mathbf{v}_{g} \leq \mathbf{v}_{g}^{\max }
$$

- generator reactive powers compatible with capability curves:

$$
\mathbf{q}_{g}^{\min } \leq \mathbf{q}_{g}(k)+\mathbf{S}_{g g} \Delta \mathbf{v}_{g}+\mathbf{S}_{g c} \Delta \mathbf{b}_{c} \leq \mathbf{q}_{g}^{\max }
$$

- shunt compensation within available limits: 


$$
\mathbf{b}_{c}^{\min } \leq \mathbf{b}_{c}(k)+\Delta \mathbf{b}_{c} \leq \mathbf{b}_{c}^{\max }
$$

Clearly, the objective of the first term in (13) is to bring pilot node voltages to their setpoint values. Assume that none of the constraints is active and the number $p$ of pilot nodes is lower than the number $g$ of controlling generators (as is the case in practice for secondary voltage control). Then, considering only the first term of the objective would yield an undetermined problem. The remaining $g-p$ degrees of freedom can be used to adjust the generator reactive powers in some optimal way. This is the purpose of the second term in (13). With $\mathbf{q}_{\varepsilon}^{\text {ref }}=\mathbf{0}$, the latter will tend to maximizing the reactive power reserves available on generators.

In the objective (13), the weighting matrices $\mathbf{P}, \mathbf{G}$ and $\mathbf{C}$ are diagonal with positive entries. The weights in $\mathbf{P}$ should be significantly larger than those in $\mathbf{G}$ so that the primary task of controlling the network voltages is achieved. The diagonal entries of $\mathbf{G}$ must also reflect the reactive power capabilities of the corresponding generators (the greater the capability, the lower the weight).

If shunt compensation is available for control, an optimization based only on the first two terms in (13) might lead to over-compensating the system, since this will relieve generators and hence decrease the second term. The purpose of the third term is precisely to prevent excessive amounts of shunt compensation to be switched on, by keeping $\mathbf{b}_{c}$ close to $\mathbf{b}_{c}^{\text {ref }}$, which would be typically set to zero. Some tuning of $\mathbf{C}$ may be required to reach this goal. Alternatively, voltage limits could be assigned to the buses where shunt compensation is available (which requires monitoring those bus voltages). Tests are needed to determine which approach is the most satisfactory.

The above optimization problem can be solved using a standard QP algorithm.

The solution so obtained defines the following moving target values of the controls:

$$
\begin{aligned}
& \mathbf{v}_{g}^{*}(k)=\mathbf{v}_{g}(k)+\Delta \mathbf{v}_{g}^{*} \\
& \mathbf{b}_{c}^{*}(k)=\mathbf{b}_{c}(k)+\Delta \mathbf{b}_{c}^{*}
\end{aligned}
$$

Note that these target values are updated at each time step $k$.

\section{Second step: dynamic reaching of targets}

In the spirit of MPC, the objective of the second step is to determine a sequence of near-future control changes corrections $\left\{\Delta \mathbf{v}_{g}(k+j), \Delta \mathbf{b}_{c}(k+j)\right\} \quad\left(j=0, \ldots, N_{c}-1\right)$ that will bring as quickly as possible the controls from their current value $\left[\mathbf{v}_{g}(k) \mathbf{b}_{c}(k)\right]$ to the moving $\operatorname{target}\left[\mathbf{v}_{g}^{*}(k) \mathbf{b}_{c}^{*}(k)\right]$. The motivation for using MPC is to produce a feasible transition (e.g. avoid switching generators under reactive limits) and compensate for modeling errors through the closed-loop structure.

To this purpose, a second optimization problem is solved whose objective is to minimize the future deviations of controls with respect to target values, i.e.

$$
J_{2}=\sum_{j=1}^{N c}\left\|\mathbf{v}_{g}^{*}(k)-\mathbf{v}_{g}(k+j)\right\|^{2}+\sum_{j=1}^{N c}\left\|\mathbf{b}_{c}^{*}(k)-\mathbf{b}_{c}(k+j)\right\|^{2}(15
$$

subject to the following constraints:

- transition from one time step to the next:

$$
\begin{aligned}
& \text { for } j=0, \ldots, N_{c}-1: \\
& \qquad \begin{array}{c}
\mathbf{v}_{g}(k+j+1)=\mathbf{v}_{g}(k+j)+\Delta \mathbf{v}_{g}(k+j) \\
\mathbf{b}_{c}(k+j+1)=\mathbf{b}_{c}(k+j)+\Delta \mathbf{b}_{c}(k+j)
\end{array}
\end{aligned}
$$

- rate of change of controls within specified range:

$$
\text { for } \begin{aligned}
j= & 0, \ldots, N_{c}-1: \\
& -\Delta \mathbf{v}_{g}^{\max } \leq \Delta \mathbf{v}_{g}(k+j) \leq \Delta \mathbf{v}_{g}^{\max } \\
& -\Delta \mathbf{b}_{c}^{\max } \leq \Delta \mathbf{b}_{c}(k+j) \leq \Delta \mathbf{b}_{c}^{\max }
\end{aligned}
$$

- generator voltages in acceptable operating ranges:

$$
\begin{aligned}
& \text { for } j=1, \ldots, N_{c}: \\
& \qquad \mathbf{v}_{g}^{\min } \leq \mathbf{v}_{g}(k+j)+\Delta \mathbf{v}_{g}(k+j) \leq \mathbf{v}_{g}^{\max }
\end{aligned}
$$

- generators reactive powers compatible with capability curves:

$$
\begin{aligned}
& \text { for } j=1, \ldots, N_{c}: \\
& \qquad \begin{array}{l}
\mathbf{q}_{g}^{\min } \leq \mathbf{q}_{g}(k+j)+\mathbf{S}_{g g} \Delta \mathbf{v}_{g}(k+j)+ \\
\qquad \mathbf{S}_{g c} \Delta \mathbf{b}_{c}(k+j) \leq \mathbf{q}_{g}^{\max }
\end{array}
\end{aligned}
$$

Other constraints could be included as well. Out of this control sequence, the first step, i.e. $\left[\Delta \mathbf{v}_{g}(k) \Delta \mathbf{b}_{c}(k)\right]$ is applied and all the procedure repeated at the next time step.

The bounds $\Delta \mathbf{v}_{g}^{\max }$ on generator voltage changes are going to be important parameters since they will dictate the rate at which voltages will be corrected. One may envisage using wider bounds (i.e. steeper changes) in emergency conditions, the latter being identified as indicated at the end of Section III.C.

We do not envisage shunt compensation as a discrete variable. Instead, we rely on the usual simplification which consists in rounding off the continuous value to the nearest discrete value. We expect that the approximation will be also compensated by the closed-loop nature of MPC. 


\section{CONCLUSION}

In this paper, several approaches to model predictive control of transmission voltages have been surveyed in the perspective of a practical application. The survey reveals growing interest, at least in the research community, in using MPC techniques. This is to be expected considering the maturity and success reported in other industrial applications as well the availability of technologies supporting its implementation in power system control centres.

MPC has indeed some potentialities but some practical issues have to be considered, and more work is needed in order to ascertain that it is viable and eventually convince practitioners to start using them.

In the light of those practical issues, a possible MPC scheme has been outlined which applies to both normal and emergency conditions. The scheme includes a static optimization to compute target control values and a dynamic optimization to produce a feasible transition. Both are repeated at each time step in order to exploit the closed-loop nature of MPC and thereby compensate for modelling errors and other sources of uncertainty.

Of course, the effective advantages of this proposed scheme remain to be carefully assessed through realistic tests.

\section{ACKNOWLEDGMENT}

The authors gratefully acknowledge the support from FNRS (Belgian National Funds for Scientific Research).

\section{REFERENCES}

[1] P. Kundur, "Power system stability and control", McGraw-Hill, 1994.

[2] S. J. Qin, T. A. Badgwell, "An overview of industrial model predictive control technology", Chemical Process Control, vol. 93, no. 316, pp. 232-256, 1997.

[3] S. J. Qin, T. A. Badgwell, "An overview of nonlinear model predictive control applications", In Nonlinear Model Predictive Control, Birkhauser Verlag, pp. 369-392, 2000.

[4] D. Q. Mayne, J. B. Rawlings, C. V. Rao, P. O. M. Scokaert, "Constrained model predictive control: Stability and optimality", Automatica, vol. 36, pp. 789-814, 2000.

[5] R. Findeisen, F. Allgower, "An introduction to nonlinear model predictive control", In Proc. 2002 Benelux Meeting on Systems and Control.

[6] M. Larsson, D. J. Hill, G. Olsson, "Emergency voltage control using search and predictive control", International Journal of Electric Power and Energy Systems, vol. 24, pp. 121-130, Jan. 2002.

[7] M. Larsson, D. Karlsson, "Coordinated system protection scheme against voltage collapse using heuristic search and predictive control", IEEE Trans. Power Systems, vol. 18, pp. 1001-1006, Aug. 2003.

[8] J. Y. Wen, Q. H. Wu, D. R. Turner, C. J. Cheng, J. Fitch, "Optimal coordinated voltage control for power system voltage stability", IEEE Trans. Power Systems, vol. 19, pp. 1115-1122, May 2004.

[9] I. A. Hiskens, B. Gong, "Voltage stability enhancement via model predictive control of load", Intelligent Automation and Soft Computing, vol. 12, no. 1, pp. 117-124, 2006.

[10] S. A. Attia, M. Alamir, C. Canudas de Wit, "Voltage collapse avoidance in power systems: a receding horizon approach", Intelligent Automation and Soft Computing, vol. 12, p. 12, 2006.

[11] M. Zima, G. Andersson, "Stability assessment and emergency control method using trajectory sensitivities", In Proc. of Bologna PowerTech, 2003.

[12] D. J. Hill, Y. Guo, M. Larsson, Y. Wang, "Global hybrid control of power systems", In Proc. of Bulk Power System Dynamics and Control $V$, Onomichi, Japan, Aug. 2001.
[13] I. A. Hiskens, B. Gong, "Voltage stability enhancement via model predictive control of load", In Proc. of Bulk Power System Dynamics and Control VI, Cortina d'Ampezzo, Italy, Aug. 2004.

[14] S. Leirens, P. Bastard, J. L. Coullon, "A hybrid approach for voltage stability of power systems", In Proc. of $15^{\text {th }}$ Power System Computation Conference, Liege, Belgium, Paper fp291, August, 2005.

[15] A. G. Beccuti, T. Geyer, M. Morari, "A hybrid system approach to power system voltage control", In Proc. of $44^{\text {th }}$ IEEE Conference on Decision and Control, Sevilla, Spain, December, 2005. [Online] Available: http://control.ee.ethz.ch

[16] T. Geyer, M. Larsson, M. Morari, "Hybrid emergency voltage control in power systems", In Proc. of European Control Conference, Cambridge, 2003.

[17] M. Zima, G. Andersson, "Model predictive control of electric power systems under emergency conditions", In Real-time stability in power systems: Techniques for early detection of the risk of blackouts, Springer, 2006.

[18] N. Atic, D. Rerkpreedapong, A. Hasanovic, A. Feliachi " NERC compliant decentralized load frequency control design using model predictive control", In Proc. of IEEE PES General Meeting, vol. 2, Toronto, 2003

[19] N. Atic, A. Feliachi, D. Rerkpreedapong, "CPS1 and CPS2 compliant wedge-shaped model predictive load frequency control", In Proc. of IEEE PES General Meeting, vol. 1, pp. 856-860, Denver, 2004

[20] A.N. Venkat, I.A. Hiskens, J.B. Rawlings and S.J. Wright, "Distributed MPC strategies for automatic generation control", In Proceedings of the IFAC Symposium on Power Plants and Power Systems Control, Kananaskis, Canada, June 2006

[21] E. Gallestey, A. Stothert, M. Antoine, S. Morton, "Model predictive control and optimization of power plant load while considering lifetime consumption", IEEE Trans. on Power Systems, vol. 17, no. 1, pp. 186191, Feb. 2002

[22] H. Vu, P. Pruvot, C. Launay, Y. Harmand, "An improved voltage control of large-scale power systems", IEEE Trans. on Power Systems, vol. 11, pp. 1295-1303, Aug. 1996.

[23] B. Marinescu, H. Bourles, "Robust predictive control for the flexible coordinated secondary voltage control of large-scale power systems", IEEE Trans. on Power Systems, vol. 14, no. 4, pp. 1262-1268, 1997.

[24] J. P. Paul, J. Y. Leost, J. M. Tesseron, "Survey of the secondary voltage control in France: present realizations and investigations", IEEE Trans. on Power Systems, vol. 2, no. 2, May 1987.

[25] V. Arcidiacono, S. Corsi, "New developments in the application of ENEL transmission system and reactive power automatic control", CIGRE 1990

[26] T. Van Cutsem, C. Moors, D. Lefebvre, "Design of load shedding schemes against voltage instability using combinatorial optimization", Proc. of the IEEE PES Winter Meeting, New York, Jan 2002, IEEE Ref $0-7803-7322-7 / 02$

[27] T. Van Cutsem, C. Vournas, "Voltage stability of electric power systems", Kluwer Academic Publisher, Dordrecht, 1998.

[28] T. Van Cutsem, M.-E. Grenier, D. Lefebvre, "Combining detailed and quasi steady-state time simulations for large-disturbance analysis", International Journal of Electric Power and Energy Systems, to appear, 2006.

[29] D. S. Popovic, V. A. Levi, Z. A. Gorecan, "Co-ordination of emergency secondary-voltage control and load shedding to prevent voltage instability", IEE Proceedings- Generation, Transmission, Distribution, vol. 144, no. 3, pp. 293-300, May 1997.

[30] I. A. Hiskens, M. A. pai, "Trajectory sensitivity analysis of hybrid systems", IEEE Trans. on Circuits and Systems-Part I, vol. 47, no. 2, pp. 204-220, Feb. 2000

[31] F. Capitanescu, T. Van Cutsem, "Unified sensitivity analysis of unstable or low voltages caused by load increases or contingencies", IEEE Trans. on Power Systems, vol. 20, pp. 321-329, 2005. 